\title{
A Numerical Accuracy Consideration in Polynomial Deflation
}

\author{
By C. J. O'Neill and T. Downs
}

\begin{abstract}
In a recent paper, Peters and Wilkinson described a composite deflation method which provides an accurate technique for the determination of the roots of a polynomial where these roots are widely spaced. By examples involving deflation by linear factors they demonstrated that the method was much more accurate than forward or backward deflation. In addition, they stated that the method could also be used when deflating by a quadratic factor or a polynomial of higher degree. In this short note it is shown that composite deflation by quadratic factors can lead to severe rounding error where two successive deflations by linear factors produce a perfectly accurate result.
\end{abstract}

A composite method of polynomial deflation has been described by Peters and Wilkinson [1] and shown to provide an accurate method of finding the roots of polynomials in situations where forward or backward deflation would fail. The method was shown to be particularly useful for polynomials with widely spaced roots. Examples were given showing deflation by linear factors, but it was stated that "exactly the same technique may be used when deflating by a quadratic factor or a factor of higher degree". This statement indicated that the method should prove useful in the inversion of polynomial or rational matrices using the method of [2] in which a considerable number of polynomial divisions are generally required. Unfortunately, on application to matrices obtained in the representation of systems with widely-spaced eigenvalues, results obtained using composite deflation were only marginally more accurate than those obtained using forward or backward deflation. It is the purpose of this short note to show that for polynomials with widely-spaced roots, composite deflation by quadratic factors or factors of higher degree may lead to severe rounding error, where deflation by linear factors leads to an accurate result. A single example should suffice.

Suppose we have a polynomial $p(x)$ given by

$$
p(x)=\left(x+10^{12}\right)\left(x+10^{6}\right)\left(x+10^{-6}\right)\left(x+10^{-12}\right) .
$$

For the purposes of illustration, we assume that we are working to four decimal digits of accuracy so that the expanded form of $p(x)$ is

$$
p(x)=x^{4}+10^{12} x^{3}+10^{18} x^{2}+10^{12} x+1 .
$$

Suppose we wish to divide $p(x)$ by the quadratic factor $q(x)$ given by

Received February 24, 1977.

AMS (MOS) subject classifications (1970). Primary 65G05; Secondary 65F05. 


$$
q(x)=\left(x+10^{12}\right)\left(x+10^{-12}\right)=x^{2}+10^{12} x+1
$$

to four decimal digits.

In such a case, the quotient should be the product of the remaining factors in $p(x)$, i.e.

$$
p(x) / q(x)=\left(x+10^{6}\right)\left(x+10^{-6}\right),
$$

so that, to four decimal digits,

$$
p(x) / q(x)=\left(x^{2}+10^{6} x+1\right) .
$$

If we divide $p(x)$ by $q(x)$ using forward deflation, we obtain

$$
x^{2}+10^{18}
$$

and using backward deflation, the result is

$$
10^{18} x^{2}+1
$$

These results show that forward deflation accurately gives the coefficient of $x^{2}$ and backward deflation accurately gives the constant term. However, because of rounding error, neither forward nor backward deflation accurately gives the coefficient of $x$, which from (1) should be $10^{6}$.

This problem does not arise if the divisor $q(x)$ is factorized and $p(x)$ divided by each factor in turn using composite deflation.

Factorizing $q(x)$ by composite deflation, we obtain

$$
q(x)=\left(x+10^{12}\right)\left(x+10^{-12}\right) .
$$

Dividing $p(x)$ by the first factor of $q(x)$ using composite deflation, we find

$$
p(x) /\left(x+10^{12}\right)=x^{3}+10^{6} x^{2}+x+10^{-6}
$$

and dividing the quotient by the remaining factor of $q(x)$ using composite deflation, we obtain

$$
\frac{p(x)}{\left(x+10^{12}\right)\left(x+10^{-12}\right)}=x^{2}+10^{6} x+1,
$$

which is the correct result (to four decimal digits).

We may conclude from the above example that composite deflation is not always accurate when deflating by quadratic factors. However, one case in which it is accurate is when the quadratic deflating factor has roots of nearly equal magnitude. This can be shown by an analysis similar to that used by Peters and Wilkinson [1] for the case of a linear deflating factor. Similar conclusions were reached in a recent paper by Bohte and Grad [3]. One way of overcoming the problem of deflation by factors of quadratic or higher degree is by factorizing the deflating factor into linear factors and dividing by each factor in turn. Composite deflation by linear factors has been applied in the inversion of a large number of polynomial and rational matrices 
and, for systems with both widely-and closely-spaced eigenvalues, the coefficients obtained in the solution were close to full accuracy.

Acknowledgement. We are grateful to a reviewer for pointing out reference [3].

Department of Electrical Engineering

University of Queensland

St. Lucia, Brisbane, Queensland, 4067

1. G. PETERS \& J. H. WILKINSON, "Practical problems arising in the solution of polynomial equations," J. Inst. Math. Appl., v. 8, 1971, pp. 16-35.

2. T. DOWNS, "On the inversion of a matrix of rational functions," Linear Algebra and Appl., v. 4, 1971, pp. 1-10.

3. Z. BOHTE \& J. GRAD, "On composite polynomial deflation by a quadratic factor," Glasnik Mat., v. 12 (32), 1977, pp. 199-208. 